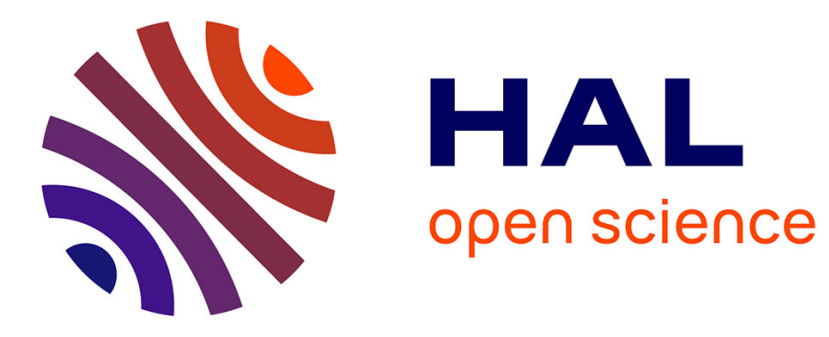

\title{
Homeomorphic Alignment of Edge-Weighted Trees
} Benjamin Raynal, Michel Couprie, Venceslas Biri

\section{To cite this version:}

Benjamin Raynal, Michel Couprie, Venceslas Biri. Homeomorphic Alignment of Edge-Weighted Trees. 7th IAPR-TC-15 International Workshop, GbRPR 2009, May 2009, Venise, Italy. pp.134143, 10.1007/978-3-642-02124-4_14. hal-01626326

\section{HAL Id: hal-01626326 https://hal.science/hal-01626326}

Submitted on 30 Oct 2017

HAL is a multi-disciplinary open access archive for the deposit and dissemination of scientific research documents, whether they are published or not. The documents may come from teaching and research institutions in France or abroad, or from public or private research centers.
L'archive ouverte pluridisciplinaire HAL, est destinée au dépôt et à la diffusion de documents scientifiques de niveau recherche, publiés ou non, émanant des établissements d'enseignement et de recherche français ou étrangers, des laboratoires publics ou privés. 


\title{
Homeomorphic Alignment of Edge-Weighted Trees
}

\author{
Benjamin Raynal, Michel Couprie, and Venceslas Biri \\ Université Paris-Est \\ Laboratoire d'Informatique Gaspard Monge, Equipe A3SI \\ UMR 8049 UPEMLV/ESIEE/CNRS
}

\begin{abstract}
Motion capture, a currently active research area, needs estimation of the pose of the subject. This requires a match between a model and the 3D shape, constructed using a multiview system. Our purpose is to realize it in real-time, using the tree representation of the skeleton of the $3 \mathrm{D}$ shape. In this paper, we propose a new alignment distance between both rooted and unrooted weighted trees, taking in account the different types of noise occuring in the data tree. Then, we develop several algorithms with acceptable time complexity for our purpose.
\end{abstract}

Key words: Graphs, homeomorphism, aligment, matching algorithm

\section{Introduction}

Motion capture without markers is a highly active research area, as shown by Moeslund and al. [1]:between 2000 and 2006, more than 350 papers on this topic were published. One difficulty of motion capture consists in finding the initial pose of the subject, represented by a 3D shape and constructed using a multiview system. Therefore, our motivation is to match the different parts of this $3 \mathrm{D}$ shape (the data) to a simple a priori model.

The model is an unrooted weighted tree (called the pattern tree), where vertices represent the different parts of the shape, and edges model the links between these parts. Edges are associated to a weight representing the distance between the parts. Concerning the data, we extract the curve skeleton of the $3 \mathrm{D}$ shape, and compute the associated weighted unrooted tree (called the data tree), by considering each multiple point and ending point, and linking them when they are directly connected. The weight of an edge is the geodesic distance between its vertices.

After this step, the main difficulty is to match the pattern tree in the data tree, with a good preservation of both topology and distances.

Several approaches have been developed, using the skeleton of a shape, in motion capture research area [2-4], and in 3D shape matching research area [57]. The best time obtained for finding the initial pose is one second [3], which is too slow, even for interactive time.

Moreover, several kinds of noise and deformities can appear in the data tree : 
Spurious branches. Due to the skeletonization algorithm and to the amount of noise of the shape surface, branchs of skeleton can appear, but without important topological signification. The method must be robust enough to work on data trees with consequent amount of spurious branches.

Useless vertex. Vertices with exactly two neighbors are not useful to describe the topology of a shape, they uselessly split an edge (and its weight) in two parts, making difficult a good matching. This kind of vertices can appear when removing spurious branches. The method must be able to match two edges joined by this kind of vertex, with a unique edge.

Splitted vertex. Vertices with more than 3 neighbors in the pattern tree can correspond to a cluster of vertices linked by weakly weighted edges in the data tree, due to the skeletonization algorithm. The method must be able to match them.

Approaches found in the literature (see Sect. 3) do not permit to achieve a robust matching, with respect to these pertubations. In the following, after reviewing basic notions, we introduce both a new alignment, called homeomorphic alignment, and a robust tree-matching algorithm which may be used for realtime pose estimation.

\section{Basics Notions}

Undirected Graphs. An undirected graph is a pair $(V, E)$, where $V$ is a finite set, and $E$ a subset of $\{\{x, y\}, x \in V, y \in V, x \neq y\}$. An element of $E$ is called an edge, an element of $V$ is called a vertex. If $\{x, y\} \in E, x$ and $y$ are said to be adjacent or neighbors. The set of all neighbors of $x$ is denoted by $\mathcal{N}(x)$. The number of vertices adjacent to a vertex $v$ is called the degree of $v$, and is denoted by $\operatorname{deg}(v)$. Let $G=(V, E)$ be an undirected graph, and let $x, y$ be in $V$, a path from $x$ to $y$ in $G$ is a sequence of vertices $s_{0}, \ldots, s_{k}$ such that $x=v_{0}, y=v_{k}$ and $\left\{v_{i-1}, v_{i}\right\} \in E, 1 \leq i \leq k$. The number $k$ is called the length of the path. If $k=0$ the path is called a trivial path. A path is closed if $x=y$. A path is simple when no vertex (except possibly $x$ ) occurs more than once in the sequence of vertices of the path. A non-trivial simple closed path in which all edges are distinct is called a cycle. A graph is connected if for all $\{x, y\} \subset V$, a path from $x$ to $y$ exists in $G$. A tree is a connected graph with no cycles. A simple path from $x$ to $y$ in a tree is unique and is denoted by $\pi(x, y)$. An unconnected graph with no cycles is called a forest, each of its connected components being a tree.

Directed Graphs. A directed graph is a pair $(V, A)$, where $V$ is a finite set, and $A$ a subset of $V \times V$. An element of $A$ is called an arc, an element of $V$ is called a vertex. Let $G=(V, A)$ be a directed graph, and let $x, y$ be in $V$, a path from $x$ to $y$ in $G$ is a sequence of vertices $s_{0}, \ldots, s_{k}$ such that $x=v_{0}, y=v_{k}$ and $\left(v_{i-1}, v_{i}\right) \in A, 1 \leq i \leq k$. The undirected graph associated to $G$ is the undirected graph $G^{\prime}=(V, E)$, such that $\{x, y\} \in E$ if and only if $(x, y) \in A$ or $(y, x) \in A$. A vertex $r \in V$ is a root of $G$ if for all $x \in V \backslash\{r\}$, a path from $r$ to $x$ in $G$ exists. $G$ is antisymetric if for all $(x, y) \in A,(y, x) \notin A$. The graph $G$ is a rooted tree 
(with root $r$ ) if $r$ is a root of $G, G$ is antisymetric and if the undirected graph associated to $G$ is a tree. An unconnected graph, where each of its connected components is a tree, is called a rooted forest.

Let $G=(V, A)$ be a rooted tree. If $(y, x) \in A$, we say that $y$ is the parent of $x$ (denoted by $\operatorname{par}(x)$ ), and that $x$ is a child of $y$. The set of all children of $y$ is denoted by $\mathcal{C}(y)$. The maximum length of a path between the root and another node is called the height of the tree. The vertices on the path from the root to a vertex $x$ are called the ancestors of $x$. We denote the set of the ancestors of $x$ by $\operatorname{anc}(x)$.

Common Definitions. Unless otherwise indicated, all the other definitions and notations in this article are similar for the two kinds of graphs. We will give them for the directed graphs, the versions for undirected graphs can be obtained by replacing arcs by edges.

Two graphs $G=\left(V_{G}, A_{G}\right)$ and $G^{\prime}=\left(V_{G^{\prime}}, A_{G^{\prime}}\right)$ are said to be isomorphic if there exists a bijection $f: V_{G} \rightarrow V_{G^{\prime}}$, such that for any pair $(x, y) \in V_{G} \times V_{G}$, $(x, y) \in A_{G}$ if and only if $(f(x), f(y)) \in A_{G^{\prime}}$.

A weighted graph is a triplet $(V, A, \omega)$, where $V$ is a finite set, $A$ a subset of $V \times V$, and $\omega$ a mapping from $A$ to $\mathbb{R}$. In a weighted tree, the weight of the unique path from $x$ to $y$, denoted by $\omega(x, y)$ is the sum of the weights of all arcs traversed in the path.

Two weighted graphs $(V, E, \omega)$ and $\left(V^{\prime}, E^{\prime}, \omega^{\prime}\right)$ are isomorphic whenever the graphs $(V, E)$ and $\left(V^{\prime}, E^{\prime}\right)$ are isomorphic.

\section{Measure of Similarity}

The problem of comparing graphs occurs in diverse areas such as computational biology, image analysis and structured databases. However, the graphs considered in these domains are most often with labeled vertices. Each notion in this section will be introduced in the case of graphs with weighted edges/arcs.

We present here measure of similarity allowing the comparison of graphs. After adapting basic edit operations for weighted trees, we define a new alignment distance: the homeomorphic alignment distance, preserving topology and handling useless and splited vertices. We then see how we handle spurious branches.

\subsection{Edit Operations}

An approach widely used to compare two graphs is to search for a sequence of simple primitive operations (called edit operations) that transforms a graph into the other and that has a minimal cost, called the edit distance.

For a graph $G=(V, A, \omega)$, commonly used operations are :

resize : Change the weight of an arc $a=(u, v) \in A$.

delete : Delete an arc $a=(u, v) \in A$ and merge $u$ and $v$ into one vertex.

insert : Split a vertex in two vertices, and link them by a new arc. 
The cost of these edit operations is given by a cost function $\gamma\left(w, w^{\prime}\right)$, where $w$ (respectively $w^{\prime}$ ) is the total weight of the arcs involved in the operation before (respectively after) its application. As a consequence, the cost of a deletion can be denoted by $\gamma(w, 0)$, where $w$ is the weight of the deleted arc, and the cost of an insertion by $\gamma(0, w)$, where $w$ is the weight of the created arc. Furthermore, we asume that $\gamma$ is a metric. Typically, $\gamma\left(w, w^{\prime}\right)=\left|w-w^{\prime}\right|$ or $\left(w-w^{\prime}\right)^{2}$.

Various edit-based distances have been defined, using different constraints on sequence order and different definitions of operations. These edit-based distances can be classified, as proposed by Wang and al. [8] : Edit distance [9], alignment distance [10,11], isolated-subtrees distance [12], and top-down distance [13]. Proposed edit distances, isolated-subtrees distances and top-down distances cannot always match all the model tree, but only subparts, most often unconnected. However, we will see in the next subsection that it is not the case for alignment distance.

\section{$3.2 \quad$ Alignment Distance}

In [10], Jiang and al. propose a similarity measure between vertex-labeled trees, that we transpose here for edge-weighted graphs.

Let $G_{1}=\left(V_{1}, A_{1}, \omega_{1}\right)$ and $G_{2}=\left(V_{2}, A_{2}, \omega_{2}\right)$ be two weighted graphs. Let $G_{1}^{\prime}=\left(V_{1}^{\prime}, A_{1}^{\prime}, \omega_{1}^{\prime}\right)$ and $G_{2}^{\prime}=\left(V_{2}^{\prime}, A_{2}^{\prime}, \omega_{2}^{\prime}\right)$ be weighted graphs obtained by inserting arcs weighted by 0 in $G_{1}$ and $G_{2}$, such that there exists an isomorphism $\mathcal{I}$ between $G_{1}^{\prime}$ and $G_{2}^{\prime}$. The set of all couples of arcs $\mathcal{A}=\left\{\left(a_{1}, a_{2}\right) ; a_{1} \in A_{1}^{\prime}, a_{2} \in\right.$ $\left.A_{2}^{\prime}, a_{2}=\mathcal{I}\left(a_{1}\right)\right\}$ is called an alignment of $G_{1}$ and $G_{2}$. The cost $C_{\mathcal{A}}$ of $\mathcal{A}$ is given by

$$
C_{\mathcal{A}}=\sum_{\left(a_{1}, a_{2}\right) \in \mathcal{A}} \gamma\left(\omega_{1}^{\prime}\left(a_{1}\right), \omega_{2}^{\prime}\left(a_{2}\right)\right) .
$$

The minimal cost of all alignments from $G_{1}$ and $G_{2}$, called the alignment distance, is denoted by $\alpha\left(G_{1}, G_{2}\right)$. Alignment distance is an interesting way in our case for three reasons:it preserves topological relations between trees, it can be computed in polynomial time, and it enables to "remove edges", regardless of the rest of the graph, solving the problem of splitted vertices.

\subsection{Homeomorphic Alignment Distance}

For the purpose of solving the useless vertex problem, we propose a new alignment, which removes 2-degree vertices.

Homeomorphism. A subdivision of an arc $(u, v)$ in a weighted graph $G=$ $(V, A, \omega)$ is an operation which consists in adding a new vertex $w$ in $V$ and two $\operatorname{arcs}(u, w)$ and $(w, v)$ in $A$, removing $(u, v)$ and assigning weights on the new arcs, such as $\omega((u, w))+\omega((w, v))=\omega((u, v))$. A subdivision of a weighted graph $G$ is a graph obtained by a sequence of subdivisions of arcs of $G$. 
The merging is the inverse operation of the subdivision, it applies only on arcs sharing a 2-degree vertex. The merging of two $\operatorname{arcs}(u, v)$ and $(v, w)$ in a weighted graph $G=(V, A, \omega)$ consists in removing $v$ in $V$, replacing $(u, v)$ and $(v, w)$ by $(u, w)$ in $A$, weighted by $\omega((u, w))=\omega((u, v))+\omega((v, w))$.

Two weighted graphs $G=\left(V_{G}, A_{G}, \omega_{G}\right)$ and $G^{\prime}=\left(V_{G^{\prime}}, A_{G^{\prime}}, \omega_{G^{\prime}}\right)$ are homeomorphic if there exists an isomorphism between some subdivision of $G$ and some subdivision of $G^{\prime}$.

Merging Kernel. Considering that a merging on a vertex $v$ on the graph $G=(V, A, \omega)$ does not affect the degree of any vertex in $V \backslash\{v\}$ (by definition of merging operation) and therefore the possibility of merging this vertex, the number of possible mergings decreases by one after each merging. In consequence, the maximal size of a sequence of merging operations, transforming $G$ into another graph $G^{\prime}=\left(V^{\prime}, A^{\prime}, \omega^{\prime}\right)$ is equal to the initial number of possible mergings in $G$. It can be remarked that any sequence of merging operations of maximal size yields the same result. The graph resulting of such a sequence on $G$ is called the merging kernel of $G$, and is denoted by $\mathcal{M K}(G)$. The following proposition is straightforward :

Proposition 1. Two graphs $G_{1}=\left(V_{1}, A_{1}, \omega_{1}\right)$ and $G_{2}=\left(V_{2}, G_{2}, \omega_{2}\right)$ are homeomorphic iff $\mathcal{M K}\left(G_{1}\right)$ and $\mathcal{M K}\left(G_{2}\right)$ are isomorphic.

Homeomorphic Alignment Distance. Let $G_{1}=\left(V_{1}, A_{1}, \omega_{1}\right)$ and $G_{2}=\left(V_{2}, A_{2}, \omega_{2}\right)$ be two weighted graphs. Let $G_{1}^{\prime}=\left(V_{1}^{\prime}, A_{1}^{\prime}, \omega_{1}^{\prime}\right)$ and $G_{2}^{\prime}=$ $\left(V_{2}^{\prime}, A_{2}^{\prime}, \omega_{2}^{\prime}\right)$ be weighted graphs obtained by deleting arcs in $G_{1}$ and $G_{2}$, such that there exists an homeomorphism between $G_{1}^{\prime}$ and $G_{2}^{\prime}$ (not necessarily unique). Let $G_{1}^{\prime \prime}=\left(V_{1}^{\prime \prime}, A_{1}^{\prime \prime}, \omega_{1}^{\prime \prime}\right)$ and $G_{2}^{\prime \prime}=\left(V_{2}^{\prime \prime}, A_{2}^{\prime \prime}, \omega_{2}^{\prime \prime}\right)$ be the merging kernels of $G_{1}^{\prime}$ and $G_{2}^{\prime}$, respectively. From proposition 1 , there exists an isomorphism $\mathcal{I}$ between $G_{1}^{\prime \prime}$ and $G_{2}^{\prime \prime}$. The set of all couples of $\operatorname{arcs} \mathcal{H}=\left\{\left(a, a^{\prime}\right) ; a \in A_{1}^{\prime \prime}, a^{\prime} \in A_{2}^{\prime \prime}, a^{\prime}=\mathcal{I}(a)\right\}$ is called an homeomorphic alignment of $G_{1}$ with $G_{2}$.

The $\operatorname{cost} C_{\mathcal{H}}$ of $\mathcal{H}$ is defined as

$$
C_{\mathcal{H}}=\sum_{\left(a, a^{\prime}\right) \in \mathcal{H}} \gamma\left(\omega_{1}^{\prime \prime}(a), \omega_{2}^{\prime \prime}\left(a^{\prime}\right)\right)+\sum_{a_{d} \in A_{1} \backslash A_{1}^{\prime}} \gamma\left(\omega_{1}\left(a_{d}\right), 0\right)+\sum_{a_{d}^{\prime} \in A_{2} \backslash A_{2}^{\prime}} \gamma\left(0, \omega_{2}\left(a_{d}^{\prime}\right)\right)
$$

This minimal cost of all homeomorphic alignments between $G_{1}$ and $G_{2}$, called the homeomorphic alignment distance, is denoted by $\eta\left(G_{1}, G_{2}\right)$.

\subsection{Cut Operation}

The last remaining problem is the presence of spurious branches, which have to be removed without any cost. For this purpose, we propose to integrate the cut operation in our alignment.

In [14], Wang et al. propose a new operation allowing to consider only a part of a tree. Let $G=(V, A, \omega)$ be a weighted tree. Cutting $G$ at an $\operatorname{arc} a \in A$, means 

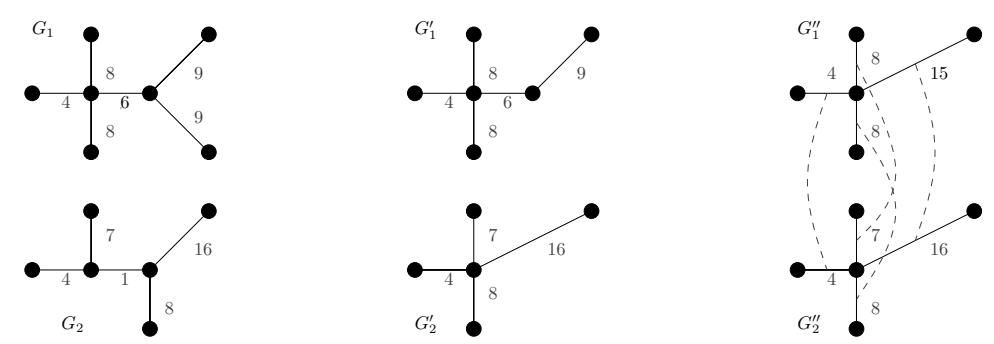

Fig. 1. Example of homeomorphic alignment: $G_{1}^{\prime}$ (resp. $G_{2}^{\prime}$ ) is obtained from $G_{1}$ (resp. $\left.G_{2}\right)$ by deletions of edges. $G_{1}^{\prime \prime}=\mathcal{M K}\left(G_{1}^{\prime}\right)$ and $G_{2}^{\prime \prime}=\mathcal{M K}\left(G_{2}^{\prime}\right)$. The dotted lines represent a possible homeomorphic alignment of $G_{1}$ and $G_{2}$, with cost equal to 12 , assuming that $\gamma(x, y)=|x-y|$.

removing $a$, thus dividing $G$ into two subtrees $G_{1}$ and $G_{2}$. The cut operation consists of cutting $G$ at an arc $a \in A$, then considering only one of the two subtrees. Let $K$ a subset of $A$. We use $C u t(G, K, v)$ to denote the subtree of $G$ containing $v$ and resulting from cutting $G$ at all arcs in $K$. In the case of a rooted tree, we consider that the root $r_{G}$ of $G$ cannot be removed by the cut operation, and then we can use the notation $\operatorname{Cut}(G, K)=\operatorname{Cut}\left(G, K, r_{G}\right)$. In the case of a rooted forest, we consider that the root of each rooted tree composing the rooted forest cannot be removed by the cut operation, and then we can use the same notation than above:Cut $(G, K)$.

Our main problem can be stated as follows:Given a weighted tree $P=$ $\left(V_{P}, A_{P}, \omega_{P}\right)$ (the pattern tree) and a weighted tree $G_{D}=\left(V_{D}, A_{D}, \omega_{D}\right)$ (the data tree), find $\eta_{\text {cut }}(P, D)=\min _{K \subseteq A_{D}, v \in V_{D}}\{\eta(P, C u t(D, K, v)\}$ (in the case of rooted trees and rooted forests, $\left.\eta_{\text {cut }}(P, D)=\min _{K \subseteq A_{D}}\{\eta(P, C u t(D, K))\}\right)$, and the associated homeomorphic alignment.

\section{Algorithms}

\subsection{Algorithm for Rooted Trees}

Let $T=(V, A, \omega)$ be a weighted tree rooted in $r_{T}$. For each vertex $v \in V \backslash\left\{r_{T}\right\}$, we denote by $\uparrow v$ the $\operatorname{arc}(w, v) \in A, w$ being the parent of $v$. We denote by $T(v), v \in V$, the subtree of $T$ rooted in $v$. We denote by $\Pi(a, b)$ the set of all vertices of the path $\pi(a, b)$. Let $v_{a}$ be an ancestor of $v$, we denote by $T_{c u t}\left(v, v_{a}\right)$ the subgraph of $T$ defined as follows :

$$
T_{\text {cut }}\left(v, v_{a}\right)=\operatorname{Cut}\left(T\left(v_{a}\right),\left\{\uparrow p^{\prime}, p^{\prime} \in \mathcal{C}(p) \backslash \Pi\left(v_{a}, v\right), p \in \Pi\left(v_{a}, \operatorname{par}(v)\right)\right\}\right) .
$$

We denote by $T\left(v, v_{a}\right)$ the tree obtained from $T_{c u t}\left(v, v_{a}\right)$ by merging on each vertex $n \in \Pi\left(v_{a}, v\right) \backslash\left\{v_{a}, v\right\}$. We denote by $\mathcal{F}(T, v)$ the rooted forest, the connected components of which are the trees $T(p, v)$, for all $p \in \mathcal{C}(v)$. By abuse 
of notation we also denote by $\mathcal{F}(T, v)$ the set of all connected components of this forest (that is, as set of trees).
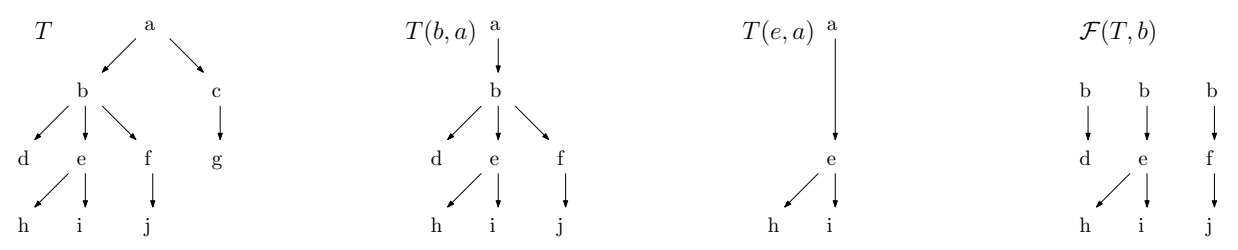

Fig. 2. Examples for a rooted tree $T$.

Proofs of the following propositions can be found in [15].

Proposition 2. Let $P=\left(V_{P}, E_{P}, \omega_{P}\right)$ and $D=\left(V_{D}, E_{D}, \omega_{D}\right)$ be two weighted trees, rooted respectively in $r_{P}$ and $r_{D}$.

$$
\eta_{\text {cut }}(P, D)=\eta_{\text {cut }}\left(\mathcal{F}\left(P, r_{P}\right), \mathcal{F}\left(D, r_{D}\right)\right) .
$$

Proposition 3. Let $i \in V_{P} \backslash\{p\}, j \in V_{D} \backslash\{d\}, i_{a} \in \operatorname{anc}(i), j_{a} \in \operatorname{anc}(j)$,

$$
\begin{aligned}
& \eta_{\text {cut }}(\emptyset, \emptyset)=0 \\
& \eta_{\text {cut }}\left(P\left(i, i_{a}\right), \emptyset\right)=\eta_{\text {cut }}(\mathcal{F}(P, i), \emptyset)+\gamma\left(\omega\left(i_{a}, i\right), 0\right) \\
& \eta_{\text {cut }}\left(\mathcal{F}\left(P, i_{a}\right), \emptyset\right)=\sum_{i^{\prime} \in \mathcal{C}\left(i_{a}\right)} \eta_{\text {cut }}\left(P\left(i^{\prime}, i_{a}\right), \emptyset\right) \\
& \eta_{\text {cut }}\left(\emptyset, D\left(j, j_{a}\right)\right)=0 \\
& \eta_{\text {cut }}\left(\emptyset, \mathcal{F}\left(D, j_{a}\right)\right)=0 .
\end{aligned}
$$

Proposition 4. Let $i \in V_{P} \backslash\{p\}, j \in V_{D} \backslash\{d\}, i_{a} \in \operatorname{anc}(i), j_{a} \in \operatorname{anc}(j)$.

$$
\begin{aligned}
& \eta_{\text {cut }}\left(P\left(i, i_{a}\right), D\left(j, j_{a}\right)\right)= \\
& \min \left\{\begin{array}{l}
\eta_{\text {cut }}(\mathcal{F}(P, i), \emptyset)+\gamma\left(\omega\left(i_{a}, i\right), 0\right) \\
\gamma\left(\omega\left(i_{a}, i\right), \omega\left(j_{a}, j\right)\right)+\eta_{c u t}(\mathcal{F}(P, i), \mathcal{F}(D, j)) \\
\min _{j_{c} \in \mathcal{C}(j)}\left\{\eta_{c u t}\left(P\left(i, i_{a}\right), D\left(j_{c}, j_{a}\right)\right)\right\} \\
\min _{i_{c} \in \mathcal{C}(i)}\left\{\eta_{\text {cut }}\left(P\left(i_{c}, i_{a}\right), D\left(j, j_{a}\right)\right)+\sum_{i_{c}^{\prime} \in \mathcal{C}(i) \backslash i_{c}} \eta_{c u t}\left(P\left(i_{c}^{\prime}, i\right), \emptyset\right)\right\} .
\end{array}\right.
\end{aligned}
$$

Proposition 5. $\forall A \subseteq \mathcal{F}(P, i), B \subseteq \mathcal{F}(D, j)$,

$$
\begin{aligned}
& \eta_{\text {cut }}(A, B)= \\
& \min \begin{cases}\min _{D\left(j^{\prime}, j\right) \in B} & \left\{\eta_{\text {cut }}\left(A, B \backslash\left\{D\left(j^{\prime}, j\right)\right\}\right)\right\} \\
\min _{P\left(i^{\prime}, i\right) \in A} & \left\{\eta_{\text {cut }}\left(A \backslash\left\{P\left(i^{\prime}, i\right)\right\}, B\right)+\eta_{\text {cut }}\left(P\left(i^{\prime}, i\right), \emptyset\right)\right\} \\
\min _{P\left(i^{\prime}, i\right) \in A, D\left(j^{\prime}, j\right) \in B}\left\{\eta_{\text {cut }}\left(A \backslash\left\{P\left(i^{\prime}, i\right)\right\}, B \backslash\left\{D\left(j^{\prime}, j\right)\right\}\right)+\right. \\
& \left.\eta_{\text {cut }}\left(P\left(i^{\prime}, i\right), D\left(j^{\prime}, j\right)\right)\right\} \\
\min _{P\left(i^{\prime}, i\right) \in A, B^{\prime} \subseteq B} & \left\{\eta_{\text {cut }}\left(A \backslash\left\{P\left(i^{\prime}, i\right)\right\}, B \backslash B^{\prime}\right)\right. \\
& \left.+\eta_{\text {cut }}\left(\mathcal{F}\left(P, i^{\prime}\right), B^{\prime}\right)+\gamma\left(\Omega\left(i^{\prime}\right), 0\right)\right\} \\
\min _{A^{\prime} \subseteq A, D\left(j^{\prime}, j\right) \in B} & \left\{\eta_{\text {cut }}\left(A \backslash A^{\prime}, B \backslash\left\{D\left(j^{\prime}, j\right)\right\}\right)+\right. \\
& \left.\eta_{\text {cut }}\left(A^{\prime}, \mathcal{F}\left(D, j^{\prime}\right) j\right)+\gamma\left(0, \Omega\left(j^{\prime}\right)\right)\right\}\end{cases}
\end{aligned}
$$


The algorithm will use a bottom-up approach: for each $(i, j) \in V_{P} \times V_{D}$ in suffix order, compute succesively $\eta_{\text {cut }}\left(P\left(i, i_{a}\right), \emptyset\right)$ and $\eta_{\text {cut }}(\mathcal{F}(P, i), \emptyset)$ (using Prop. 3 ), $\eta_{\text {cut }}(A, B), \forall A \subseteq \mathcal{F}(P, i), B \subseteq \mathcal{F}(D, j)$ (using Prop. 5), and $\eta_{\text {cut }}\left(P\left(i, i_{a}\right), D\left(j, j_{a}\right)\right), \forall i_{a} \in \operatorname{anc}(i), j_{a} \in \operatorname{anc}(j)$ (using Prop.4). Then, return $\eta_{\text {cut }}(P, D)$, using Prop.2.

The total computation time complexity is in $O\left(\left|V_{P}\right| *\left|V_{D}\right| *\left(2^{d_{P}} * 2^{d_{D}} *\left(d_{D} *\right.\right.\right.$ $\left.\left.2^{d_{P}}+d_{P} * 2^{d_{D}}\right)+h_{P} * h_{D} *\left(d_{P}^{2}+d_{D}\right)\right)$, where $d_{G}$ and $h_{G}$ denote, respectively, the maximal degree of a vertex in $G$ and the height of $G$. If the maximal degree is bounded, the total computation time complexity is in $O\left(\left|V_{P}\right| *\left|V_{D}\right| * h_{P} * h_{D}\right)$.

\subsection{Algorithm for Unrooted Trees}

Let $G=(V, E, \omega)$ be a weighted tree, let $r \in V$, we denote by $G^{r}$ the directed weighted tree rooted in $r$, such that $G$ is the undirected graph associated to $G^{r}$.

Proposition 6. Let $P=\left(V_{P}, E_{P}, \omega_{P}\right)$ and $D=\left(V_{D}, E_{D}, \omega_{D}\right)$ be two weighted trees.

$$
\eta_{c u t}(P, D)=\min _{i \in V_{P}, j \in V_{D}}\left\{\eta_{c u t}\left(P^{i}, D^{j}\right)\right\} .
$$

Let $P=\left(V_{P}, E_{P}, \omega_{P}\right)$ and $D=\left(V_{D}, E_{D}, \omega_{D}\right)$ be two undirected weighted trees. We denote by $\mathcal{F}(P, a, b), a, b \in V_{P}$ the set of rooted trees $P^{r}, r \in V_{P}$, such that $b$ is an ancestor of $a$ in $P^{r}$. We denote by anc $(P, a, b), a, b \in V_{P}$ the set of the ancestors of $a$ in at least one rooted tree in $\mathcal{F}(P, a, b)$. We denote by $\mathcal{C}(P, a, b), a, b \in V_{P}$ the set of the children of $a$ in at least one rooted tree in $\mathcal{F}(P, a, b)$.

Our algorithm is based on an approach similar to bottom-up. It is easy to see that for computing $\eta_{\text {cut }}\left(P^{p}\left(i, i_{a}\right), D^{d}\left(j, j_{a}\right)\right)$ and $\eta_{c u t}\left(\mathcal{F}\left(P^{p}, i\right), \mathcal{F}\left(D^{d}, j\right)\right)$, for any $p \in V_{P}, d \in V_{d}$ we need to know $\eta_{c u t}\left(P^{p}\left(i_{c}, i\right), D^{d}\left(j_{c}, j\right)\right)$ for all $i_{c} \in$ $\mathcal{C}(P, i, p), j_{c} \in \mathcal{C}(D, j, d)$. We start by computing $\eta_{\text {cut }}\left(P^{p}\left(i, i_{a}\right), D^{d}\left(j, j_{a}\right)\right)$ and $\eta_{\text {cut }}\left(\mathcal{F}\left(P^{p}, i\right), \mathcal{F}\left(D^{d}, j\right)\right.$ ), for all $i$ (respectively $j$ ) being a leaf of $P^{p}$ (respectively $\left.D^{d}\right)$ and continue iteratively with vertices which have all their children already computed.

The total computation time of this algorithm is in $O\left(\left|V_{P}\right| *\left|V_{D}\right| *\left(d_{P} *\right.\right.$ $\left.\left.2^{d_{P}+2 * d_{D}}+d_{D} * 2^{d_{D}+2 * d_{P}}+\left|V_{P}\right| *\left|V_{D}\right| *\left(d_{P}^{2}+d_{D}\right)\right)\right)$ complexity. If the maximal degree is bounded, the total computation is in $O\left(\left|V_{P}\right|^{2} *\left|V_{D}\right|^{2}\right)$ time complexity.

\section{Experimentation}

\subsection{Usage of Homeomorphic Alignment}

In case of motion capture, we can use homeomorphic alignment in three different ways :

- between the two unrooted trees, if we have no a priori knowledge.

- between two rooted trees, obtained from the unrooted trees by rooting them on vertices we want to match together.

- between a rooted tree and an unrooted tree, if we want to be sure that the root is conserved by the homeomorphic alignment. 


\subsection{Results}

Our model tree contains seven vertices, representing head, torso, crotch, the two hands and the two feet. Experimentally, the data tree obtained from the skeleton of the visual hull has a degree bounded by 4 , and its number of vertices is between seven and twenty, with a gaussian probability repartition centred on ten. All the results have been obtained on a computer with a processor Xeon 3 $\mathrm{GHz}$ and 1 Go of RAM.

For finding the average time of computation of our algorithm, we have randomly generated 32 pattern trees, and for each pattern tree, we have generated 32 data trees, which yields 1024 pairs of trees. Each pattern tree has seven vertices, one of which has a degree equals to 4 . Each data tree has at least one 4-degree vertex. The results of the four kinds of alignments are shown on Fig. 3.

In the average case $\left(\left|V_{D}\right| \leq 12\right)$, the homeomorphic alignement between a rooted pattern tree and a unrooted data tree (we assume than the torso is always aligned), can be easily computed in real time (frequence superior to $24 \mathrm{~Hz}$ ) and in the worst case $\left(\left|V_{D}\right| \simeq 20\right.$ ), we keep an interactive time (frequence superior to $12 \mathrm{~Hz}$ ). For tracking, if we can use the homeomorphic alignment between two rooted trees, we are widely above $50 \mathrm{~Hz}$.

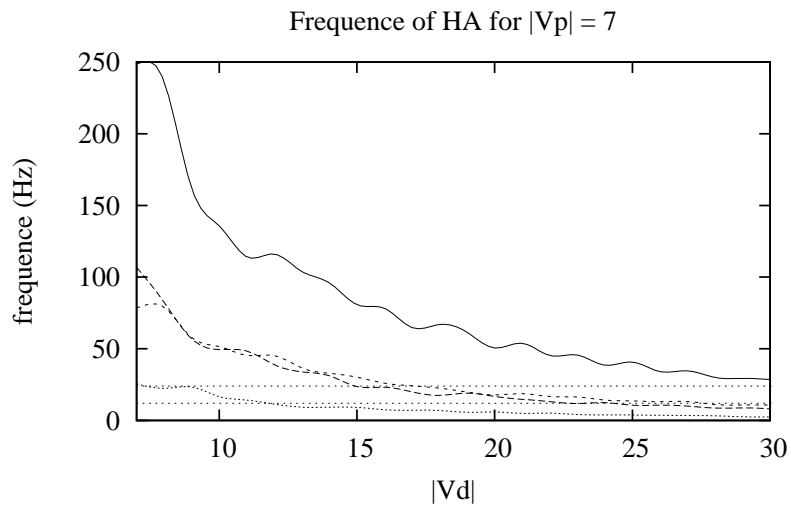

HA(rooted P,rooted D) HA(rooted P, D)

$\mathrm{HA}(\mathrm{P}$, rooted $\mathrm{D})$

$\mathrm{HA}(\mathrm{P}, \mathrm{D})$ real time interactive time

Fig. 3. Frequences of the differents homeomorphic alignments for variable sizes of data tree.

\section{Conclusion}

In this paper, we have introduced a new type of alignment between weighted trees, the homeomorphic aligment, taking into account the topology and avoiding the noise incluced by spurious branches, splited and useless 2-degree vertices. We have also developed several robust algorithms to compute it with a good complexity, which enable its application in real time for motion capture purpose. 
In future works, we will take into account more useful information on the model, such as spatial coordinates of data vertices, and include them in our algorithm, for a better robustness. Finally, using this alignment, we will propose a new fast method of pose initialization for motion capture applications.

\section{References}

1. Moeslund, T.B., Hilton, A., Krüger, V.: A Survey of Advances in Vision-based Human Motion Capture and Analysis. In: Computer Vision and Image Understanding vol. 104(2-3), pp. 90-126. Elsevier (2006)

2. Chu, C., Jenkins, O., Mataric, M.: Markerless Kinematic Model and Motion Capture from Volume Sequences. In: IEEE Computer Society Conference on Computer Vision and Pattern Recognition. vol 2., IEEE Computer Society (2003)

3. Menier, C., Boyer, E., Raffin, B.: 3d Skeleton-based Body Pose Recovery. In: Proceedings of the 3rd International Symposium on 3D Data Processing, Visualization and Transmission, Chapel Hill (USA). (2006)

4. Brostow, G., Essa, I., Steedly, D., Kwatra, V.: Novel Skeletal Representation for Articulated Creatures. LNCS, pp. 66?78. Springer, Heidelberg (2006)

5. Sundar, H., Silver, D., Gagvani, N., Dickinson, S.: Skeleton Based Shape Matching and Retrieval. In SMI, pages 130-139, (2003)

6. Baran, I., Popović, J.: Automatic rigging and animation of 3D characters. In: International Conference on Computer Graphics and Interactive Techniques, ACM Press New York, NY, USA (2007)

7. Cornea, N., Demirci, M., Silver, D., Shokoufandeh, A., Dickinson, S., Kantor, P.: 3D Object Retrieval using Many-to-many Matching of Curve Skeletons. In: Shape Modeling and Applications. (2005)

8. Wang, J., Zhang, K.: Finding similar consensus between trees: an algorithm and a distance hierarchy. Pattern Recognition 34(1) pp. 127-137. Elsevier (2001)

9. Tai, K.: The Tree-to-Tree Correction Problem. Journal of the ACM 26(3) pp. 422?433. ACM New York, NY, USA (1979)

10. Jiang, T., Wang, L., Zhang, K.: Alignment of Trees - an Alternative to Tree Edit. In: CPM 94: Proceedings of the 5th Annual Symposium on Combinatorial Pattern Matching, London, UK, pp. 75-86. Springer-Verlag (1994)

11. Jansson, J., Lingas, A.: A Fast Algorithm for Optimal Alignment between Similar Ordered Trees. LNCS vol. 2089 pp. 232-??. Springer, Heidelberg (2001)

12. Tanaka, E., Tanaka, K.: The Tree-to-tree Editing Problem. International Journal of Pattern Recognition and Artificial Intelligence. 2(2) pp. 221-240 (1988)

13. Selkow, S.: The Tree-to-Tree Editing Problem. Information Processing Letters 6(6) pp. 184-186 (1977)

14. Wang, J., Zhang, K., Chang, G., Shasha, D.: Finding Approximate Patterns in Undirected Acyclic Graphs. In : Pattern Recognition vol.35(2) pp. 473?483. Elsevier (2002)

15. Raynal, B., Biri, V., Couprie, M.: Homeomorphic Alignment of Weighted Trees. Internal report IGM 2009-01. LIGM, Université Paris-Est (2009) 